\title{
DEFICIÊNCIA DE BORO EM MAMONA (1)
}

\author{
EDISON MARTINS PAULO (2), ONDINO CLEANTE BATAGLIA $(3,4)$, \\ FRANCISCO SEIITI KASAI $(2,4)$ E JOSÉ CARLOS CAVICHIOLI (2)
}

\begin{abstract}
RESUMO
No ano agrícola 1987/88, na Estação Experimental de Agronomia da Alta Paulista em Adamantina (SP), foram observados, após um período de seca, em plantas de mamona (Ricinus communis L.) var. IAC-80, sintomas de enrugamento das folhas, necrose do ápice de racemos primários, redução do número de frutos e necrose no caule. Após o reinicio das chuvas, as plantas com sintomas, comparadas às sem sintomas, tinham concentraçóes mais elevadas de todos os nutrientes, porém apresentavam valores semeIhantes de boro. Amostras de solo coletadas sob plantas deficientes tinham concentraçōes mais baixas de boro do que sob plantas normais. Os dados disponiveis e a semelhança com sintomas observados em outras plantas levaram à conclusão que aqueles estão provavelmente associados à deficiência de boro.
\end{abstract}

Termos de indexação: mamona, Ricinus communis $L$., deficiência de boro, seca.

A mamona (Ricinus communis L.) é uma fonte praticamente pura de ácido ricinoléico, que, além da ampla gama de aplicação industrial é fonte alternativa de combustivel, possibilitando tornar a cultura importante elemento econômico e estratégico do País (SAVY FILHO \& BANZATO, 1987). O Brasil é o

(1) Trabalho recebido para publicação em 13 de junho e aceito em 26 de setembro de 1989.

(2) Estaçāo Experimental de Agronomia da Alta Paulista, Instituto Agronómico, Caixa Postal 28, 13001 Campinas, SP.

(3) Seção de Fertilidade do Solo e Nutrição de Plantas, IAC.

(4) Com bolsa de pesquisa do CNPq. 
maior produtor mundial dessa oleaginosa, destacando-se, em São Paulo, a regiāo oeste, pela considerável área de plantio.

No ano agrícola 1987/88, na Estaçāo Experimental de Agronomia da Alta Paulista, do Instituto Agronômico, em latossolo vermelho-escuro, textura média, observou-se o desenvolvimento anormal de plantas de mamona var. IAC-80, caracterizado inicialmente por internódios curtos e pelo enrugamento e enrolamento. do limbo foliar, similarmente a algumas viroses. Esses indicios começaram a aparecer quando as plantas tinham cerca de 45 dias, depois da aplicação da primeira adubação nitrogenada em cobertura, realizada com $30 \mathrm{~g} / \mathrm{cova}$ de sulfato de amônio, seguida por um periodo de 18 dias de estiagem. Posteriormente, apareceram também manchas negras no caule, correspondentes à necrose interna dos vasos condutores, necrose progressiva do ápice para a base nos racemos primários em inicio de formação, superbrotamento e queda abundante de folhas mais velhas. Os racemos primários, quando formados, apresentavam número reduzido de frutos - Figuras 1 e 2 . Quadro sintomático similar foi encontrado na cultura da batata (Solanum tuberosum L. subsp. tuberosum Hawkes) (HIROCE et al., 1971) e do algodão (Gossypium hirsutum L.) (SILVA et al., 1979, 1982), sendo relacionado com a deficiência de boro.

$\mathrm{Na}$ tentativa de esclarecer o problema, coletaram-se ao acaso, dentro da população, plantas anormais e plantas consideradas normais, submetendo-as à determinaçāo de macronutrientes e micronutrientes, conforme os métodos descritos por BATAGLIA et al. (1983). Analisaram-se delas os seguintes órgãos: (a) caule; (b) folhas; (c) racemo primário em inicio de formação, e (d) racemo primário formaco. A partir desses resultados - Quadro 1 - calcularam-se as relaçóes entre as concentraçōes de boro e macronutrientes para cada parte das plantas consideradas - Quadro 2. Amostras de solo foram coletadas na profundidade de $0-20 \mathrm{~cm}$ e na projeção da copa das plantas normais e anormais, obtendo-se duas amostras compostas: estas foram analisadas pelos métodos descritos por RAIJ \& QUAGGIO (1983) para fins de avaliação de fertilidade e de boro solúvel em água quente, extraido na proporção 1:2 (solo:soluçāo) e determinado pelo método colorimétrico da azometina $\mathrm{H}$. Esses resultados encontram-se no quadro 3.

Comparando-se, no quadro 1 , as plantas com e sem sintomas, observa-se que as anormais apresentavam, de modo geral, nos diversos órgãos examinados, concentraçōes mais elevadas de quase todos os nutrientes, enquanto no boro tais valores eram bastante semelhantes. Isso pode ser explicado por ter a amostragem sido feita cerca de 45 dias após o aparecimento do quadro sintomático descrito, quando o período de estiagem já havia terminado e a mamona, retomado o desenvolvimento. Dessa forma, as plantas anormais, em função do menor crescimento, acumularam os nutrientes na parte aérea, o que não aconteceu com o boro, provavelmente por estar deficiente. Isso pode ser visto mais claramente no quadro 2, pelas relações entre os teores de boro e macronutrientes: nas plantas com sintomas, o boro ocorreu em proporçōes mais baixas do que nas normais. 

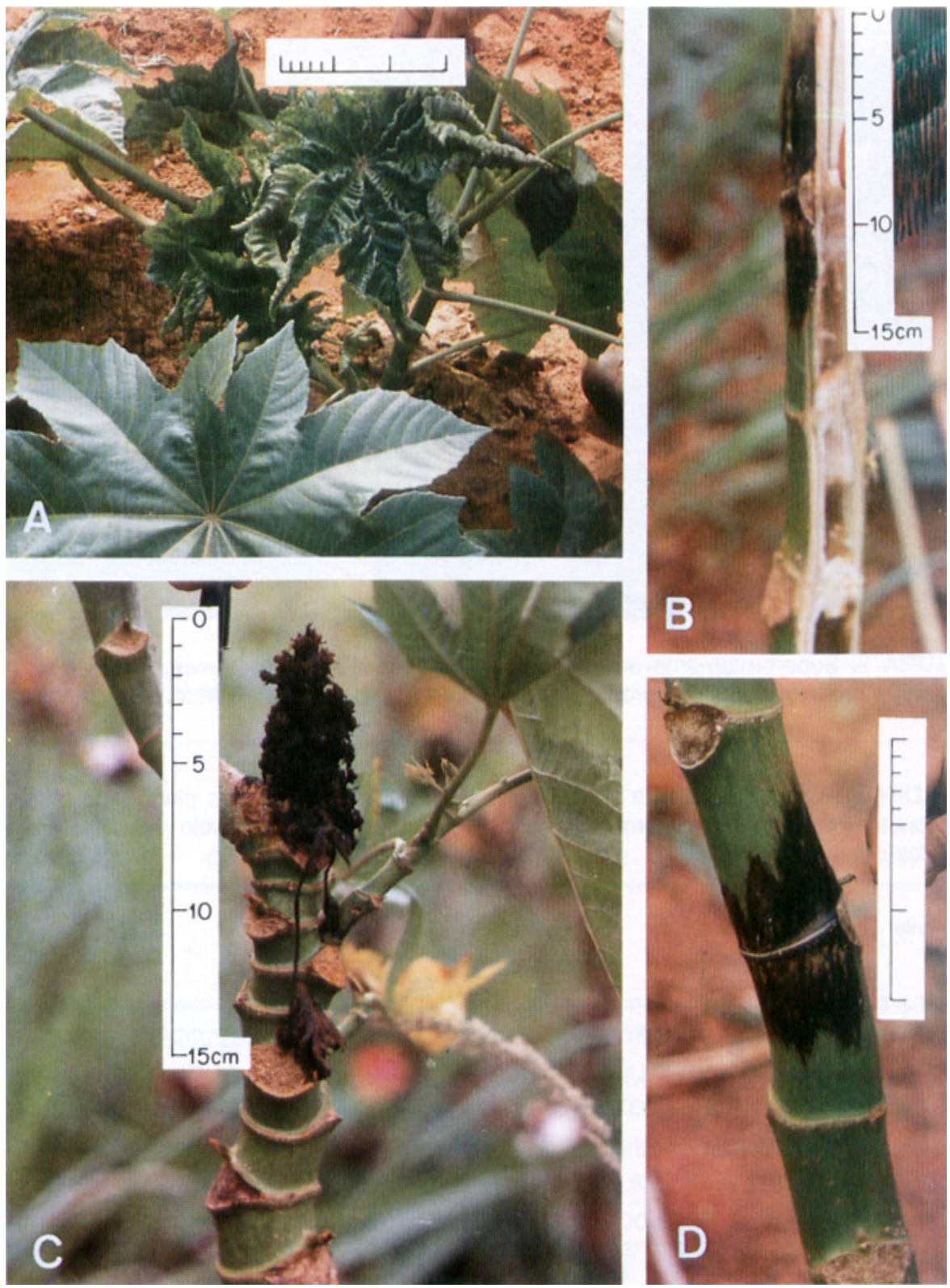

FIGURA 1. Sintomas de deficiência de boro em mamoneira caracterizados por enrolamento das folhas $(A)$, manchas negras no caule com necrose interna dos vasos condutores $(B$ e $D)$, internódios curtos e necrose dos racemos primários $(C)$. 


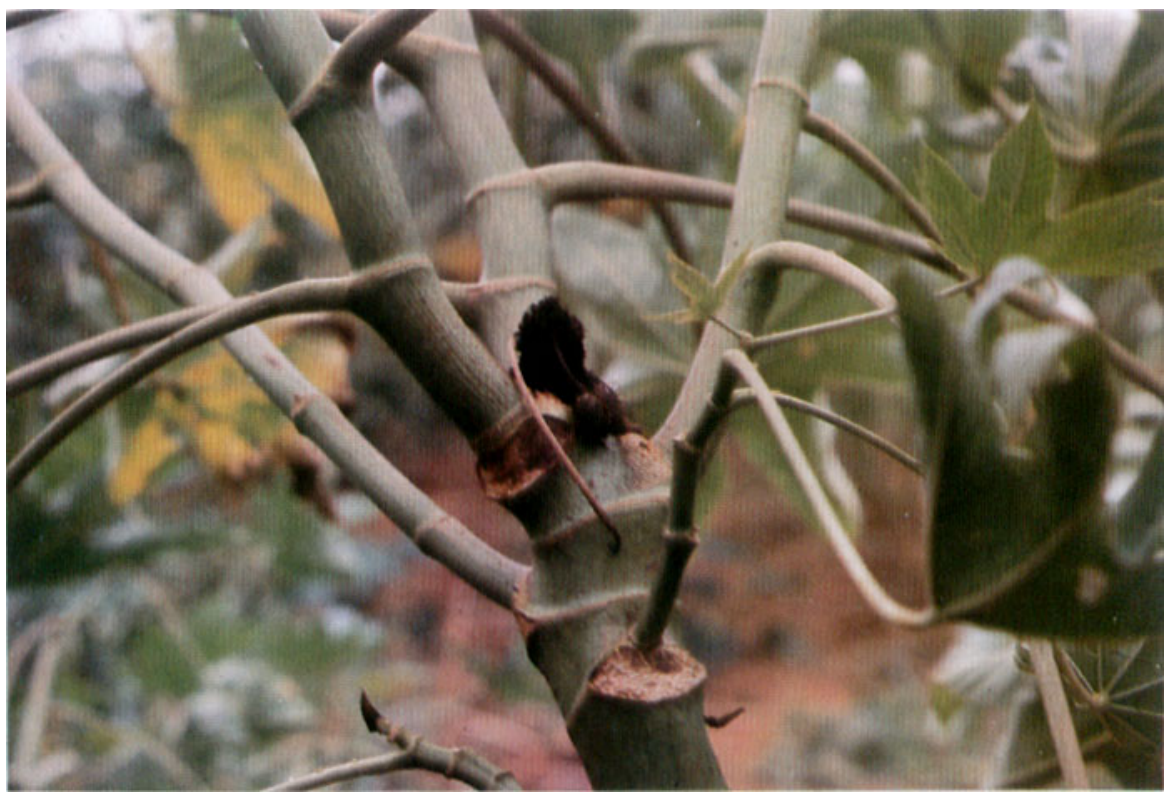

FIGURA 2. Superbrotamento em mamoneira, após o inicio das chuvas, em plantas que sofreram deficiência de boro em período anterior, sob estresse hídrico.

QUADRO 1. Concentração de nutrientes nos diferentes órgãos das plantas de mamona com sintoma (CS) e sem sintoma (SS), vegetando em latossolo vermelho-escuro, textura média

\begin{tabular}{|c|c|c|c|c|c|c|c|c|}
\hline \multirow[t]{2}{*}{ Nutriente } & \multicolumn{2}{|c|}{ Caule } & \multicolumn{2}{|c|}{ Folha } & \multicolumn{2}{|c|}{ Racemo } & \multicolumn{2}{|c|}{$\begin{array}{l}\text { Racemo em início } \\
\text { de formaçāo }\end{array}$} \\
\hline & $\mathrm{CS}$ & SS & CS & SS & $\operatorname{cs}$ & SS & $\mathrm{CS}$ & SS \\
\hline$N$ & 1,330 & 0,820 & 4,290 & 3,290 & 4,120 & 3,170 & 4,870 & 3,950 \\
\hline$P$ & 0,251 & 0,118 & 0,445 & 0,247 & 0,420 & 0,327 & 0,555 & 0,464 \\
\hline K & 3,710 & 2,440 & 3,560 & 3,270 & 3,220 & 2,630 & 3,710 & 3,320 \\
\hline $\mathrm{Ca}$ & 0,800 & 0,750 & 1,790 & 1,530 & 0,520 & 0,570 & 1,400 & 0,800 \\
\hline $\mathrm{Mg}$ & 0,270 & 0,230 & 0,560 & 0,230 & 0,340 & 0,280 & 0,530 & 0,420 \\
\hline $\mathrm{s}$ & 0,136 & 0,149 & 0,286 & 0,232 & 0,216 & 0,175 & 0,294 & 0,245 \\
\hline$B$ & 22 & 18 & 56 & 50 & 27 & 31 & 30 & 30 \\
\hline $\mathrm{Cu}$ & 3,0 & 2,0 & 7,8 & 5,4 & 7,5 & 5,7 & 7,1 & 7,7 \\
\hline $\mathrm{Fe}$ & 33 & 29 & 67 & 65 & 46 & 46 & 81 & 50 \\
\hline$M n$ & 75 & 66 & 272 & 139 & 73 & 70 & 111 & 77 \\
\hline $\mathrm{Zn}$ & 20,6 & 16,5 & 40,1 & 21,7 & 36,4 & 23,5 & 54,4 & 42,3 \\
\hline
\end{tabular}


QUADRO 2. Relação entre a concentração de boro e macronutrientes nos órgāos ḍas plantas de mamona com sintoma (CS) e sem sintoma (SS)

\begin{tabular}{|c|c|c|c|c|c|c|c|c|}
\hline \multirow[t]{2}{*}{ Nutriente } & \multicolumn{2}{|c|}{ Caule } & \multicolumn{2}{|c|}{ Folha } & \multicolumn{2}{|c|}{ Racemo } & \multicolumn{2}{|c|}{$\begin{array}{l}\text { Racemo em inicio } \\
\text { de formação }\end{array}$} \\
\hline & CS & SS & $\mathrm{CS}$ & SS & $\mathrm{CS}$ & SS & $\mathrm{CS}$ & SS \\
\hline$N$ & 16 & 22 & 13 & 15 & 7 & 10 & 6 & 8 \\
\hline$P$ & 88 & 152 & 126 & 202 & 64 & 96 & 54 & 65 \\
\hline$k$ & 6 & 7 & 16 & 15 & 8 & 12 & 8 & 9 \\
\hline $\mathrm{Ca}$ & 27 & 24 & 31 & 33 & 52 & 54 & 21 & 38 \\
\hline $\mathrm{Mg}$ & 82 & 78 & 100 & 217 & 79 & 111 & 57 & 71 \\
\hline$s$ & 162 & 121 & 196 & 215 & 125 & 177 & 102 & 122 \\
\hline
\end{tabular}

QUADRO 3. Resultados das análises químicas do solo amostrado na projeção da copa de plantas de mamona com e sem sintoma

\begin{tabular}{|c|c|c|c|c|c|c|c|c|c|}
\hline $\begin{array}{l}\text { Amostragem } \\
\text { sob plantas }\end{array}$ & $P$ & MO & $\begin{array}{c}\mathrm{pH} \\
\mathrm{CaCl}_{2}\end{array}$ & K & $\mathrm{Ca}$ & $\mathrm{Mg}$ & $H+A l$ & v & $\mathrm{B}$ \\
\hline & $\mu \mathrm{g} / \mathrm{cm}^{3}$ & $\%$ & & $\ldots$ & $\mathrm{meq} / 100 \mathrm{~cm}^{3}$ & & - & $\%$ & ppm \\
\hline Com sintomas & 8 & 1,3 & 4,7 & 0,32 & 0,7 & 0,4 & 2,0 & 41 & 0,16 \\
\hline Sem sintomas & 21 & 0,8 & 4,4 & 0,18 & 0,9 & 0,2 & 2,2 & 37 & 0,20 \\
\hline
\end{tabular}

As análises do solo - Quadro 3 - revelaram menor teor de boro no solo das plantas com sintomas, vindo a corroborar a interpretação dos resultados das determinações da parte aérea da mamona, embora, de acordo com SIQUEIRA et al. (1987), ambos os valores sejam classificados como teores médios.

A ocorrência da sindrome após a adubação nitrogenada em cobertura e de estiagem está em acordo com o relatado por MALAVCLTA (1987) e LOPES (1987), que mencionam que tais situações, em terras pobres em boro, acentuam a deficiência desse nutriente sobre as plantas cultivadas.

No ano agricola 1988/89, implantando-se campo de multiplicaçāo de mamona var. IAC-80 com o uso da fórmula 4-14-8 + boro e havendo regime pluviométrico nomal, não se observou o quadro sintomático. Entretanto, sugere-se experimentação em soluçāo nutritiva ou em solos deficientes para confirmação dos sintomas caracterizados neste trabalho como deficiência de boro. 


\section{SUMMARY}

\section{BORON DEFICIENCY IN CASTOR BEAN PLANTS}

During the summer season 1987/88, castor bean plants (Ricinus communis L.) var. IAC-80 growing at the Estaçâo Experimental de Agronomia da Alta Paulista, State of São Paulo, Brazil, showed some symptomus related to boron deficiency. The symptoms included leaf curling, necrosis of terminal bud, small fruit setting and necrosis in the stem. The symptoms occurred after a drought period. Plants were analysed after a new raining period. The nutrient concentrations were higher in plants with deficiency symptoms except for boron. The relations betwen boron and each one of the macronutrients were lower in plants with symptoms. Soil samples collected under deficient plants were lower in boron than samples collected under normal plants. Based on these informations and on the similarity with boron deficiency symptoms on other plants, it was conclued that the symptoms might be related to boron deficiency.

Index terms: castor bean, Ricinus communis L., boron deficiency, drought.

\section{REFERÉNCIAS BIBLIOGRÁFICAS}

BATAGLIA, O.C.; FURLANI, A.M.C.; TEIXEIRA, J.P.F.; FURLANI, P.R. \& GALLO, J.R. Métodos de análise química de plantas. Campinas, Instituto Agronômico, 1983. 48p. (Boletim técnico, 78)

HIROCE, R.; GALLO, J.R. \& NÓBREGA, S.A. Deficiência de boro em batatinha cultivada em solo orgânico do Vale do Pairaiba. Bragantia, Campinas, 30:V-VII, 1971. (Nota, 2)

LOPES, A.S. Micronutrientes: dinâmica e disponibilidade. In: FERNANDES, F.M. \& NASCIMENTO, V.M., coord. Curso de atualização em fertilidade do solo. Campinas, Fundação Cargill, 1987. p.209-246.

MALAVOLTA, E. Nutrição mineral de plantas. In: FERNANDES, F.M. \& NASCIMENTO, V.M., coord. Curso de atualização em fertilidade do solo. Campinas, Fundação Cargill, 1987. p.33-101.

RAIJ, B. van \& QUAGGIO, J.A. Métodos de análise do solo para fins de fertilidade. Campinas, Instituto Agronôrnico, 1983. 31p. (Boletim técnico, 81)

SAVY FILHO, A. \& BANZATO, N.V. Mamona. In: Campinas, Instituto Agronômico. Instruçôes ayricolas para o Estado de São Paulo, editado por Mário José Pedro Júnior, E.A. Bulisani; C.V. Pommer; F.A. Passos; I.J. Godoy \& C. Aranha. Campinas, 1987. p.231. (Boletim, 200) 
SILVA, N.M.; CARVALHO, L.H.; BATAGLIA, O.C. \& HIROCE, R. Efeitos do boro em algodoeiro cultivado em condiçōes de casa de vegetação. Bragantia, Campinas, 38:153164, 1979.

; CHIAVEGATTO, E.J.; SABINO, N.P. \& HIROCE, R. Efeito de doses de boro aplicadas no sulco de plantio do algodoeiro em solo deficiente. Bragantia, Campinas, 41:181-191, 1982.

SIQUEIRA, O.J.F.; SCHERER, E.E.; TASSINARI, G.; ANGHINONI, I.; PATELLA, J.F.; TEDESCO, M.J.; MILAN, P.A. \& ERNANI, P.R. Recomendaçôes de adubação e calagem para os Estados do Rio Grande do Sul e Santa Catarina. Passo Fundo, EMBRAPA/CNPT, 1987. 100p. 